\title{
Motor Fluency Deficits in the Sequencing of Actions in Schizophrenia
}

\author{
Yvonne Delevoye-Turrell \\ Université de Lille III
}

\author{
Alan M. Wing \\ University of Birmingham
}

\author{
Anne Giersch \\ Université L. Pasteur of Strasbourg \\ Jean-Marie Danion \\ Université L. Pasteur of Strasbourg
}

\begin{abstract}
Many everyday activities depend on the capacity to organize and smoothly execute motor sequences. The authors tested the hypothesis that a sequencing deficit is associated with schizophrenia. They used a new method to distinguish between lower and higher order mechanisms for the impairment. The 1st task involved triggered sequences in which sensory information from 1 movement was the cue for initiation of the following movement. Results showed that the motor sequences were performed as fluently in patients as in controls. The 2 nd and 3rd tasks involved sequences in which the entire movement sequence could be preplanned. Patients executed the sequences less fluently than controls but only under the condition where action sequences were required. Furthermore, the patients' fluency deficit increased with sequence complexity. The high discrimination power of Task 3 gave the authors a means to control for a potential psychometric confound involving differential discriminating power and to argue in favor of a specific higher order motor fluency deficit in patients with schizophrenia. It is suggested that basic lower order mechanisms that integrate sensory information with outgoing motor commands are preserved in schizophrenia, whereas higher order integrative mechanisms that are required for the smooth coordination of motor sequences are impaired.
\end{abstract}

Keywords: schizophrenia, motor sequencing, grip force, planning, reaction time

The act of lifting and manipulating an object is a commonly performed motor action that is nevertheless a complex process involving close temporal coordination of activity both in the proximal arm muscles responsible for lifting and in the finger muscles used to grip the object (Flanagan \& Wing, 1997). In control subjects, grip force is tightly scaled both to the mass and to the texture of manipulated objects (Johansson \& Westling, 1984). In patients with schizophrenia, even if finger forces are scaled both to texture and mass (Delevoye-Turrell, Giersch, \& Danion, 2003), excessive and unnecessarily high force levels are developed (Caligiuri \& Lohr, 1994; Neumann \& Walker, 1999; Vrtunski, Simpson, \& Meltzer, 1989). Recent work suggests, however, that this problem of force efficiency may arise only when action sequencing is required (Delevoye-Turrell et al., 2003). For example, in a reach-and-lift task patients with schizophrenia exhibited a significant delay between the onset of grip closure and the start of the upward lift movement; such an uncoordinated force pattern may

Yvonne Delevoye-Turrell, EA 1059 URECA, Université de Lille III, Villeneuve d'Ascq, France; Anne Giersch and Jean-Marie Danion, UMR-S 666, Unité Psychiatrique, Université L. Pasteur of Strasbourg, Strasbourg, France; Alan M. Wing, Behavioural Brain Science Centre, University of Birmingham, Birmingham, United Kingdom.

This research was supported by the Alliance program and the United Kingdom Medical Research Council. The study was conducted in the psychiatry unit of the University Hospitals of Strasbourg.

Correspondence concerning this article should be addressed to Yvonne Delevoye-Turrell, EA 1059 URECA, Université de Lille III, Rue du Barreau BP419, Villeneuve d'Ascq 59360, France. E-mail: yvonne.delevoye@free.fr have led to the development of excessive force levels in the patients compared with the control subjects. This abnormal behavior is consistent with the hypothesis that schizophrenia involves a fundamental cognitive abnormality, or dysmetria (Andreasen, 1999), which would be characterized by a deficit in the fluent coordination of sequences of action and thought (Crespo Facorro, Paradiso, Andreasen, \& O'Leary, 1999; Wiser et al. 1998).

The aim of the present study was to test directly the hypothesis that schizophrenia is characterized by a problem in the fluent coordination of motor sequences and to discriminate between a lower order deficit (i.e., the integration of afferent sensory input with efferent motor output) and a higher order deficit (i.e., the programming of motor sequences). Furthermore, we sought to distinguish between those processes involved in planning and execution and those involved in coordinating multiple motor elements within a single sequence.

In a task where a single effector is used to perform a sequence, each successive submovement-or motor element-is dependent on completion of the preceding one. It takes an appreciable amount of time for peripheral sensory information to reach the central nervous system, and in the presence of such delays, it is generally suggested that motor sequences may be organized in two fundamentally different ways depending on whether or not they require sensory information (for a review, see Delevoye-Turrell \& Wing, 2004). First, sequencing may be based on concurrent processing of sensory information (Macefield \& Johansson, 1994). In this case, each element in the sequence will be initiated only after the arrival of the sensory feedback signaling the end of the preceding element. This mechanism will result in significant time intervalsapproximately $90 \mathrm{~ms}$ (Johansson, Riso, Häger, \& Bäckström, 
1992) - between the completion of one element and the initiation of the next element of the sequence. In the following, we refer to motor actions organized in this fashion as triggered sequences. An alternative approach to sequencing involves preplanning the entire sequence before its initiation as a whole (Billon, Semjen, Cole, \& Gauthier, 1996). In this case, the sequence is represented as a single entity and performed without reference to feedback from events within the sequence. These motor sequences are referred to in the following as planned sequences. The correct outcome of these planned sequences relies on well-defined predictive processes developed through previous experience as well as the ability to organize through time and to coordinate information from multiple sources (e.g., context or memory based information). Over a series of trials, optimization of motor fluency for both triggered and planned sequences is reached by minimizing the time delays between successive elements.

In order to gain a better understanding of the nature of the motor sequencing deficits that may underlie schizophrenia (e.g., Sullivan et al., 1994), we evaluated motor fluency for both triggered and planned sequences in 24 patients with schizophrenia by means of three contrasting motor tasks.

\section{Task 1: Triggered Sequences}

The fluent execution of an action might be impaired in schizophrenia because sensory information is not well integrated with outgoing motor commands (Gordon \& Soechting, 1995) because of dysfunctional lower order integrative processes of a subcortical (cerebellar) nature (Müller \& Dichgans, 1994). We tested this hypothesis by means of a triggered sequencing task, during which subjects were required to let an object slip within grasp and then quickly augment grip force in order to arrest object slip. Here, grip augmentation (second element) could be initiated only after the arrival of sensory afferences from the digits signaling object slip (first element). Thus, a successful trial required the fluent integration of afferent and efferent information about hand function.

Previously used in microneurographic studies, this task has provided the means to assess the role of the mechanoreceptors of the glabrous skin for rapid and accurate afferences about discrete mechanical events, for example, changes in shear force or slip on the skin (Johansson \& Weslting, 1987). These studies have shown that triggered responses are abolished both in deafferented patients (Rothwell et al., 1982) and during anesthetic block (Johansson, Häger, \& Riso, 1992; Nowak, Glasauer, Meyer, Mai, \& Hermsdorfer, 2002), stressing the importance of afferent information for triggered actions. In addition, as the time course for triggered actions is of the order of $100 \mathrm{~ms}$, the underlying neural mechanisms are very probably reflexive, possibly involving cortical and subcortical pathways (Harrison, Mayston, \& Johansson, 2000; Johansson, Lemon, \& Westling, 1994; Macefield \& Johansson, 1994), because the latencies are too short to involve controlled, voluntary intervention (Johansson \& Westling, 1984). Consequently, we propose that this task can provide a simple method for the quantitative study of lower order integrative processes required for the fluent integration of incoming sensory information regarding slip with the outgoing motor program about grip.

\section{Task 2: Planned Sequences}

To test the possibility that motor sequencing deficits previously described in patients with schizophrenia might be due to a problem in higher order integrative processes, we used a sequential pointing task for which the timing of all elements of the sequence was self-initiated as well as self-paced. Consequently, the entire sequence could be preplanned as a single movement (Sternberg, Knoll, Monsell, \& Wright, 1989). Here, a single two-element tapping motion (finger down; finger up) was incorporated within a set of motor sequences whose complexity was increased. The critical variable measured was the duration of the brief contact between the index finger and the top surface of a target object. This paradigm was specifically designed to reproduce the problem in prolonged contact duration described above in patients with schizophrenia during the reach-to-lift task (Delevoye-Turrell et al., 2003).

Here, both lower order and higher order integrative processes are required for normal performance. Indeed, lower order mechanisms are needed to minimize the duration of the brief contact (through the fluent integration of afferent and efferent information). In addition, higher order processes are needed to integrate the tapping motion within sequences of increasing complexity.

\section{Task 3: Context Effect}

Finally, to distinguish a problem in motor coordination from a more general deficit in motor planning, we used a simple visuomotor pointing task; through instruction, the emphasis was set either on minimizing execution time or on minimizing both initiation and execution time. Under the first condition, emphasis was set upon the endpoint of the reach only, encouraging subjects to consider the task as a single movement of one element (point). Under the second condition, subjects were encouraged to first focus on removing the hand from a touch pad as fast as possible and then quickly continue through with the pointing task. Because the task required subjects to divide their attention between starting and executing the action, we suggest that the instruction created an arbitrary segmentation of the reach into two elements (lift and point).

In order to be fast, motor planning was necessary under both conditions. Thus, if schizophrenia affects motor planning, then patients should reveal abnormal patterns of response under both conditions. However, if the deficit lies within the integrative mechanisms required for the coordination through time of multiple subelements of a sequence, then patients should be specifically impaired under the condition where the arbitrary segmentation of the motor action was introduced.

In summary, our approach was to measure the time delay between successive motor elements both in triggered (Task 1) and in planned motor sequences (Tasks 2 and 3) in order to examine the possibility that schizophrenia affects higher order mechanisms - those required for the fluent execution of planned sequences. Task 3 was designed specifically to distinguish between a deficit affecting integration and a more general problem in motor planning.

\section{Method}

\section{Participants}

Twenty-four outpatients with schizophrenia (14 men; mean age $=36.0 \pm 7.5$ years; mean educational level $=10.1 \pm 3.3$ years) and 24 healthy control subjects $(14$ men; mean age = 
$35.4 \pm 11.9$ years; mean educational level $=13.7 \pm 4.6$ years $)$ participated in a single experimental session that lasted $60 \mathrm{~min}$. It was composed of three different motor tasks. Subjects and patients were paid for their participation. Written informed consent was obtained in accordance with the recommendations of the Declaration of Helsinki. The experimental protocol was approved by the local ethics committee.

Psychiatric diagnoses of patients (9 disorganized, 7 characterized with positive symptoms, and 8 characterized with negative symptoms) were established by a senior psychiatrist in agreement with the patients' therapist through a semi-structured interview (the Mini International Neuropsychiatric Interview; Lecrubier et al., 1997); patients were included in the study only if they fulfilled the Diagnostic and Statistical Manual of Mental Disorders (4th ed.; American Psychiatric Association, 1994) criteria for schizophrenia. The psychiatrist collected the demographic information and ran the series of clinical tests for all subjects. The average age at symptom onset was $24.6 \pm 8.4$ years, the average disease duration was $12.8 \pm 8.0$ years, and the number of hospitalization periods was $6.1 \pm 6.6$. None of the patients had received any changes in their treatment regimens over the preceding 3 months, suggesting that they were clinically stable. Mean symptom severity, as measured by the Brief Psychiatric Rating Scale total score, was $37.0 \pm 12.8$. With the Scale for the Assessment of Positive Symptoms and the Scale for the Assessment of Negative Symptoms, the group mean scores were $20.0 \pm 18.2$ and $34.6 \pm 24.6$, respectively. Of the 24 patients, 21 were receiving atypical neuroleptic treatment administered in a standard dose (mean dose $=$ $181.0 \pm 90.1 \mathrm{mg} /$ day of chlorpromazine or equivalent). Eight were also receiving an antiparkinsonian treatment (mean dose $=13.7$ $\mathrm{mg} /$ day; range $=5-30 \mathrm{mg}$ /day of lepticur equivalent). Three of the patients were not receiving any treatment.

Control subjects were recruited through local advertising and were pair-matched with the patients with schizophrenia. There were no significant differences between controls and patients for age, sex, or level of education $(p>.40)$. Exclusion criteria for both groups were visual and auditory disorders, history of neurological illness or trauma, alcohol and substance abuse, and age older than 55 years or younger than 18 years. Subjects were all right-handed as determined through the Edinburgh Handedness Inventory.

\section{Apparatus}

We used a six-axis load cell (ATI-Novatech Gamma SI-13010) to measure force (in N) and torque (in N.mm ${ }^{-1}$ ) changes in $x$-, $y$-, and $z$-axes. In Tasks 2 and 3, we taped an accelerometer (Entran EGA-F-25) to the top of the subjects' hand and recorded hand acceleration (in $\mathrm{m}_{\mathrm{s}} \mathrm{s}^{-2}$ ) during hand movement. Data were sampled at a $1000 \mathrm{~Hz}$.

\section{Tasks}

We used three motor tasks to determine the nature of the motor sequencing problems in schizophrenia.

\section{Task 1: Triggered Sequences}

Subjects were seated in front of a table and used the right dominant hand to grip the load cell in precision grip with thumb on one side and index, middle, and ring fingers on the other. The load cell was held with the $x$-axis aligned vertically, the $y$-axis aligned horizontally parallel with the grip axis, and the $z$-axis aligned horizontally perpendicular to the grip axis. The subjects held this object approximately $10 \mathrm{~cm}$ above the tabletop. Their task was to release their grip on the manipulated object in order to allow it to slip under the effects of gravity. As soon as the load cell began to slip, their task was to arrest the fall of the object by increasing the grip force applied through the fingers (for more details about the procedure and apparatus, see Turrell, Li, \& Wing, 2001). In order for a trial to be accepted, slips were required to occur within $3 \mathrm{~s}$ from the start of the trial, which was signaled by an auditory signal. A block of 10 trials was recorded for each individual.

\section{Task 2: Planned Sequences}

The load cell was placed flat on the table $20 \mathrm{~cm}$ in front of the subjects' right-dominant shoulder. The accelerometer was used to record hand acceleration during movement. Two series of trials were run in an identical set order for all subjects. The first series was intended to measure the tap duration in a natural situation (i.e., without subjects paying attention to the dynamic characteristics of the tap); the second series explicitly put pressure on the subjects to minimize contact duration.

1. Natural condition. Subjects produced series of five taps on the top of the load cell. They were required to produce these taps with the index finger and without moving the wrist, so that the palm of the hand remained in contact with the table. From one series to the next, they were encouraged to change the rhythm of execution of the sequence. At the end of each series, subjects were asked to reproduce verbally the rhythm they had just executed (e.g., "dit dah dah dit dit"). This procedure was followed to encourage subjects to pay attention to the entire sequence and not to the way in which they executed each tap. Subjects performed 10 trials. The contact duration of only the first tap of each sequence was analyzed and was considered to comprise two elements (2elm: finger down and finger up).

2. Pressure condition. In this condition, subjects were explicitly instructed to produce the shortest tap possible on the top of the load cell. Twenty trials were performed for each type of sequence, in a blocked semirandomized order. In the 2-elm sequence, subjects tapped the top of the load cell with the index finger without moving the wrist so that the palm of the hand remained in contact with the table. In the three-element (3-elm) sequence, subjects started each trial with the hand resting on a touch pad that was at the edge of the table aligned with their right shoulder. The subjects' task was to reach toward and tap the top of the load cell. Finally, in the four-element (4-elm) sequence, subjects started with the wrist resting on the touch pad and their task was to reach toward the load cell, tap the top of it, then lift the hand up to stabilize it precisely $30 \mathrm{~cm}$ above the tabletop. Visual indication was provided beyond the object to indicate the target zone for the end of the movement. For all three sequences, the subjects were reminded every five trials that the goal of the tapping action was to produce the shortest contact duration possible on the load cell.

\section{Task 3: Context Effect}

As in the previous task, the load cell was placed flat on the table $20 \mathrm{~cm}$ in front of the subjects' right-dominant shoulder. For all 
trials, subjects rested the wrist of the right dominant hand on the initial touch pad, which was fixed at the edge of the table. At the auditory signal, the subjects' task was to move the hand to tap the top surface of the load cell with the index finger. There were two experimental conditions. In the first, subjects were instructed to tap the load cell as fast as possible at the auditory signal (global sequences, 1-elm). In the second, subjects were required to lift the hand off the touch pad as fast as possible at the auditory signal. This instruction was intended to encourage subjects to be both fast in initiating and fast in executing the movement (segmented sequences, 2-elm).

\section{Results}

\section{Task 1: Triggered Sequences}

We measured baseline levels during the first $250 \mathrm{~ms}$ of each trial. The start of object vertical slip along the $x$-axis produced a change in torque about the $y$-axis. Slip onset was identified as the time at which the torque about this $y$-axis first showed a change greater than five times the variability of the baseline. The start of grip force increase was taken as the first moment in time when the rate of change of grip force was five times greater than the standard deviation of the baseline. We ran a $t$ test $^{1}$ to assess the effect of group (controls, patients) on mean grip force baseline, mean force stability, and mean time delays between the start of object vertical slip and the start of grip force increase.

The slip task requires the use of sensory feedback about slip onset to arrest object fall by increasing grip force. All subjects required practice in order to succeed in letting the object slip within $3 \mathrm{~s}$ from the start of the trial. On average, patients required more practice trials than controls (eight vs. two trials). Nevertheless, the general pattern of slip/grip force modulation was similar for all subjects (Figure 1). The object was dropped only five times in total across the 48 subjects. It was not dropped more often by patients than by controls.

The analysis of the experimental trials revealed that patients used higher grip force levels $(M=5.6 \mathrm{~N})$ and revealed greater force instability $(S D=1.2 \mathrm{~N})$ than matched controls $(M=5.3 \mathrm{~N}$, $S D=0.8 \mathrm{~N}$ ), but these differences did not reach significance. For the mean time delays, the group effect did not reach significance, $F(1,46)=-0.015, n s$. The time between object slip and the start of grip force increase was $94 \mathrm{~ms}(S D=18 \mathrm{~ms})$ and $95 \mathrm{~ms}(S D=$ $21 \mathrm{~ms}$ ) for controls and patients, respectively (see left side of Figure 2). These results suggest that the fluent integration of afferent and efferent information for hand function is preserved in schizophrenia. Mean time intervals were similar for the nontreated (98 $\mathrm{ms}, S D=29 \mathrm{~ms}$ ) and the treated $(93 \mathrm{~ms}, S D=20 \mathrm{~ms}$ ) patients with schizophrenia, suggesting no secondary effect of treatment on lower order motor processes.

\section{Task 2: Planned Sequences}

For each subject and experimental condition, we measured the force and contact duration of the taps. Overall, patients applied higher force levels $(7.2 \mathrm{~N}$; range $=1.1-28.0 \mathrm{~N})$ than the controls $(3.8 \mathrm{~N}$; range $=1.5-8.3 \mathrm{~N}), F(1,46)=-5.103, p<.05$. For both groups, contact durations were constant across the force range: The force-duration correlation coefficients were not significantly different from zero for either the patients or the controls, which

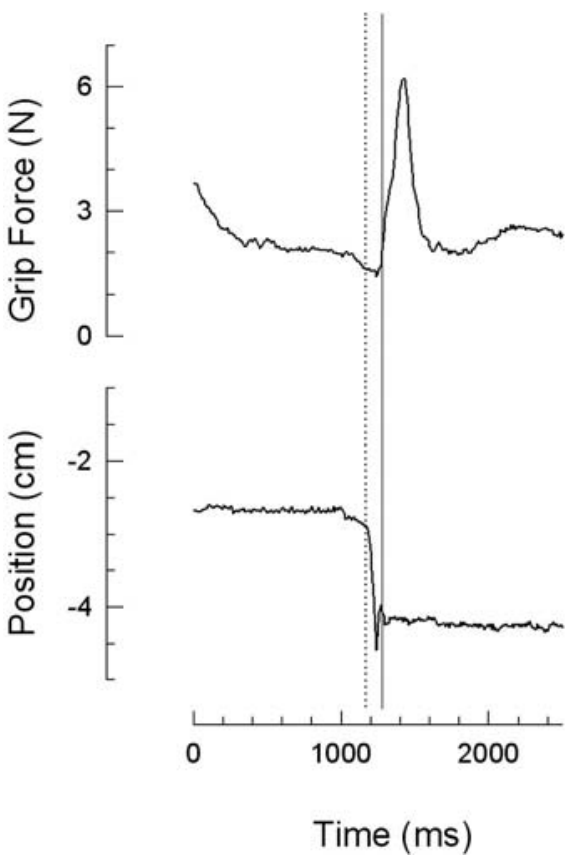

Figure 1. A typical trial from the triggered sequence task as seen in a typical medicated patient with schizophrenia. The subjects' task was to slowly release grip on the object (top section) in order to let the object slip by a few centimeters (bottom section) without letting it drop. As soon as they detected the occurrence of a finger slip (dotted line), subjects typically increased grip force (solid line) in order to arrest object fall. The force patterns were similar in patients and pair-matched controls.

suggested that the tapping duration was not dependent on the finger force exerted at contact. Finally, the force levels were equivalent across the four types of sequences for both patients and controls. Consequently, in the following, only the contact duration of the taps are reported.

First, we carried out a two-way repeated measures analysis of variance (ANOVA) with Group (controls, patients) and Condition (natural: 2-elm; pressure: 2-elm) as factors in order to assess the effect of emphasizing the importance of a short contact duration. Second, we carried out a two-way repeated measures ANOVA on the results from the pressure condition only in order to test the effect of sequence complexity with Group (controls, patients) and Complexity (2-elm, 3-elm, 4-elm) as factors. Finally, we subjected the magnitudes of peak acceleration and peak deceleration to a two-way repeated measures ANOVA with Group (controls, patients) and Complexity (3-elm, 4-elm) as factors.

Patients and controls performed all sequences without apparent difficulty. The first ANOVA (natural vs. pressure) revealed a significant main effect of group, $F(1,46)=42.561, p<.001$ : The contact duration between finger and object was shorter for controls $(M=44 \mathrm{~ms}, S D=18 \mathrm{~ms})$ than for patients $(M=116 \mathrm{~ms}, S D=$ $63 \mathrm{~ms})$. The condition main effect was also significant, $F(1,46)=$ $11.993, p<.01$, indicating that when emphasis was set on minimizing the contact duration, the contact durations were shortened significantly for both patients (from $134 \mathrm{~ms}[S D=67 \mathrm{~ms}]$ to 98

\footnotetext{
${ }^{1}$ All statistical analyses were two-sided and interpreted in relation to a significance level set at $\alpha=.05$.
} 


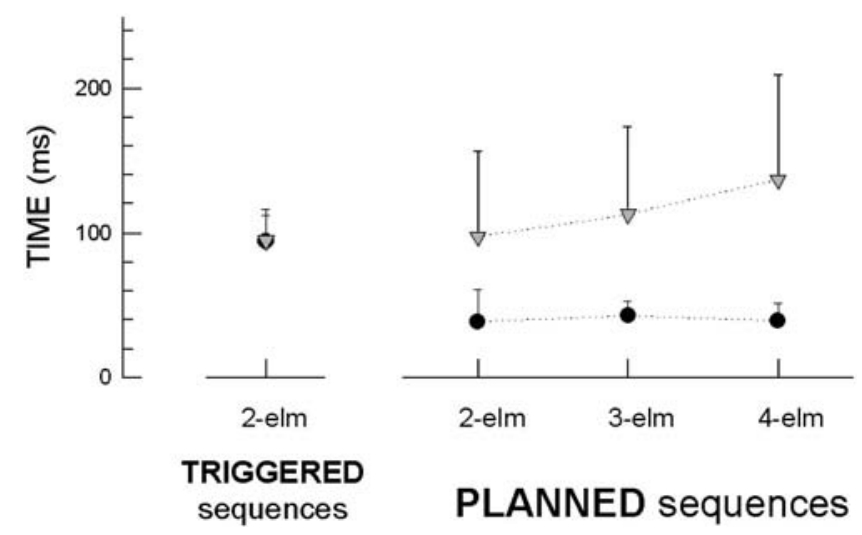

Figure 2. Mean contact durations (with standard deviations) for controls and patients with schizophrenia. This measure is an indicator of the programmed time interval between the end of the first element and the start of the second element of a sequence as a function of the nature (triggered sequences; planned sequences) and the complexity of the sequences (2elm, 3-elm, 4-elm). Results are reported for movements requiring subjects to sequence two elements (2-elm), three elements (3-elm), or four elements (4-elm).

$\mathrm{ms}[S D=59 \mathrm{~ms}]$ ) and controls (from $50 \mathrm{~ms}[S D=15 \mathrm{~ms}]$ to 39 $\mathrm{ms}[S D=22 \mathrm{~ms}])$. The Group $\times$ Condition interaction was not significant, $F(1,46)=3.365$, ns.

For the sequence complexity manipulation, an ANOVA revealed again a main effect of group, $F(1,46)=141.989, p<.001$. The Group $\times$ Complexity interaction was also significant, $F(2$, $92)=20.998, p<.001$. The Tukey honestly significant difference (HSD) test indicated that for controls, contact durations were similar for all sequences. For the patients, the more complex the sequence, the longer were the contact times (see right side of Figure 2). This effect of sequence complexity on the mean time of contact was present in both the nontreated $(85,112,137 \mathrm{~ms})$ and the treated $(100,129,136 \mathrm{~ms})$ patients with schizophrenia.

The kinematic profiles were similar in the 3-elm and the 4-elm sequences for both patients and controls. However, the duration of the reaching movement was longer for patients $(M=608 \mathrm{~ms}$, $S D=141 \mathrm{~ms})$ than for controls $(M=521 \mathrm{~ms}, S D=135 \mathrm{~ms}), F(1$, $46)=9.499, p<.01$. An ANOVA revealed that controls $(M=$ $0.39 \mathrm{~m} . \mathrm{s}^{-2}$; range $\left.=0.17-0.61 \mathrm{~m} . \mathrm{s}^{-2}\right)$ accelerated significantly faster than patients $\left(M=0.29 \mathrm{~m} \cdot \mathrm{s}^{-2}\right.$; range $\left.=0.11-0.48 \mathrm{~m} \cdot \mathrm{s}^{-2}\right)$, $F(1,46)=3.201, p<.05$. However, both groups decelerated at a similar rate (combined $M=0.42 \mathrm{~m} . \mathrm{s}^{-2} ;$ range $=0.26-0.57 \mathrm{~m} . \mathrm{s}^{-2}$ ), $F(1,46)=0.503$, ns. These results suggest that overall patients and controls used a similar visuomotor guidance strategy to precisely position the hand over the load cell before initiating the tap motion: For all subjects, the index finger contacted the object (i.e., the downward motion of the finger was terminated) before complete immobilization of the hand.

\section{Task 3: Context Effect}

Figure 3 illustrates the mean reaction times obtained for the control group (black circles) and the patient group (white triangles) under the two experimental conditions (global sequences: 1-elm; segmented sequences: 2-elm). An ANOVA revealed a significant Group $\times$ Condition interaction, $F(1,46)=6.199, p<.01$. The Tukey HSD test showed that the reaction times for global sequences were similar for controls $(M=282 \mathrm{~ms}, S D=131 \mathrm{~ms})$ and for patients $(M=292 \mathrm{~ms}, S D=63 \mathrm{~ms}), p=.62$. However, for segmented sequences, reaction times were significantly faster in controls $(M=250 \mathrm{~ms}, S D=59 \mathrm{~ms})$ than in patients $(M=342 \mathrm{~ms}$, $S D=121 \mathrm{~ms}), p<.001$. Finally, controls reacted significantly faster for segmented than for global sequences (difference of 32 $\mathrm{ms}$ ), whereas patients reacted slower for segmented than for global sequences (difference of $49 \mathrm{~ms}$ ). Nontreated patients reacted faster than treated patients, but performance patterns were similar in both groups (295 vs. $254 \mathrm{~ms}$ in the nontreated patients and 350 vs. 297 $\mathrm{ms}$ in the treated patients, for the segmented and the global conditions, respectively).

For movement times, the group effect was significant, $F(1$, $46)=24.77, p<.001$ : Patients moved overall slower $(M=346$ $\mathrm{ms}, S D=7 \mathrm{~ms})$ than controls $(M=310 \mathrm{~ms}, S D=8 \mathrm{~ms})$. Both groups moved faster for global $(M=259 \mathrm{~ms}, S D=8 \mathrm{~ms})$ than for segmented sequences $(M=405 \mathrm{~ms}, S D=8 \mathrm{~ms}), F(1,46)=$ $430.89, p<.001$, as encouraged through instruction. The interaction was significant, $F(1,46)=16.49, p<.001$, indicating that the increase in movement time between the global and the segmented sequences was greater for controls (from $221 \mathrm{~ms}[S D=8 \mathrm{~ms}$ ] to $402 \mathrm{~ms}[S D=8 \mathrm{~ms}]$ ) than for patients (from $287 \mathrm{~ms}[S D=7 \mathrm{~ms}]$ to $408 \mathrm{~ms}[S D=7 \mathrm{~ms}])$. Movement times were similar for the nontreated $(M=329 \mathrm{~ms}, S D=195 \mathrm{~ms})$ and the neuroleptictreated patients $(M=348 \mathrm{~ms}, S D=133 \mathrm{~ms})$.

\section{Evaluating a Potential Psychometric Confound}

Experimental psychopathology struggles with the fact that patients' behavioral impairments are difficult to interpret as specific cognitive deficits. This difficulty, referred to as the psychometric

\section{- Control subjects $(\mathrm{N}=24)$ Patients with schizophrenia ( $\mathrm{N}=24)$}

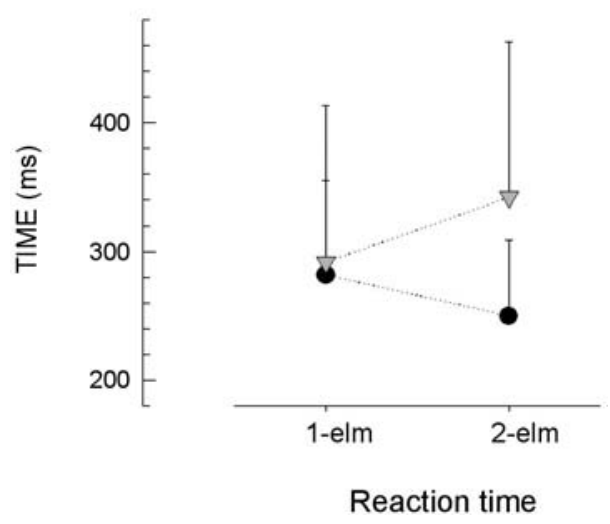

Figure 3. Mean group reaction times (with standard deviations) for controls and patients with schizophrenia performing the pointing task under pressured-time conditions emphasizing the execution time (global condition: 1-elm) or both the start and the execution time (segmented condition: 2-elm) of the pointing movement. 1-elm = one element in task; 2-elm $=$ two elements in task. 
confound (MacDonald \& Kang, 2006), occurs because mental disorders such as schizophrenia usually result in impairments across many tasks, and some of these impairments may appear larger simply because a task has greater discriminating power (Chapman \& Chapman, 1973). Hence, in Table 1, we present four indicators that together provide the information needed to argue here in favor of a specific problem in the fluent coordination of planned sequences in patients with schizophrenia, rather than simply reflecting differential sensitivity in the tests used.

First, we computed Cronbach's alpha (the reliability coefficient; Cronbach, 1951) for the control group only in order to provide a measure of internal consistency for each task and condition (see Table 1). One can note that, except for the 2-elm condition of Task 3 , the alpha values are similar across and acceptable for all tasks and conditions as they are greater than .70 (Miller, 1995; Nunnaly, 1978). Second, we calculated the discriminating power, which is indexed by true score variance (TSV), for each task and condition as the product of the observed score variance and the reliability value (see Table 1). Here, results showed that Task 3 possesses the highest TSV, whereas Task 1 is where it is the lowest.

The existence of a group effect in Task 2 but not in Task 1 could have been attributed to task differences in TSV coupled with a generalized deficit in patients. However, the inclusion of Task 3 provides the means to discuss the specificity of the reported deficits. Indeed, our results reveal that the global (1-elm) condition of Task 3 has greater TSV than any condition of Task 1 or 2 but yields no group difference. Following, for example, Strauss (2001) and Knight and Silverstein (2001), the fact that the TSVs are ranked differently than the group differences suggests a specific fluency problem rather than a generalized deficit.

Finally, to evaluate the group effect size of the tasks where a group difference was found, we computed Cohen's $d$. Results (Table 1, far right column) show that Task 1, Task 3, and Task 2 are increasingly powerful to discriminate between patients and controls. Following Cohen and Servan-Schreiber (1992), the protocol used in Task 2 provides a large effect size because $d>0.8$, and the 2-elm condition of Task 3 would provide a medium effect size with $d>0.5$.

\section{Discriminant Validity}

In a final analysis, we computed the intercorrelation values among tasks and conditions for the control group only in order to assess task specificity. Results are presented in Table 2 and reveal that the three conditions in Task 2 are highly correlated with each other (boldface values). However, correlations are near zero between Task 1 and Task 2, suggesting that the triggered and the planned sequencing tasks very probably tap processes that are not related to each other, that is, that are different. We suggest that Task 1 (triggered sequences) and Task 2 (planned sequences) reflect the working state of lower and higher order integrative processes, respectively, with only the latter being impaired in patients with schizophrenia for the fluent sequencing of action sequences.

\section{Discussion}

The aim of this study was to test the hypothesis that schizophrenia is characterized by a problem in the fluent coordination of motor sequences and to discriminate between lower and higher order mechanisms for the impairment. Our results confirm a fluency problem in schizophrenia but only for the execution of planned motor sequences, that is, those sequences requiring functional higher order processes. Similar observations were made in treated and nontreated patients, suggesting that the fluency deficits for planned sequences may be a core impairment in schizophrenia.

In our first task (triggered sequences), the incoming sensory information from the tips of the fingers (object slip; first element of the sequence) had to be used to readjust the force level applied (grip increase; second element of the sequence). Hence, successful performance of the task was possible only through the fluent integration of afferent and efferent aspects of hand function, in

Table 1

Comparison of Control Group Scores and Patient Group Scores on Tasks 1, 2, and 3

\begin{tabular}{|c|c|c|c|c|c|c|c|c|}
\hline \multirow{2}{*}{$\begin{array}{l}\text { Task \& } \\
\text { condition }\end{array}$} & \multicolumn{2}{|c|}{ Control group } & \multicolumn{2}{|c|}{ Patient group } & \multirow{2}{*}{$\begin{array}{c}\text { Reliability } \\
\text { (Cronbach's } \alpha \text { ) }\end{array}$} & \multirow{2}{*}{$\begin{array}{c}\text { Discrimination } \\
\text { power (TSV) }\end{array}$} & \multirow{2}{*}{$\begin{array}{c}\text { Group } \\
\text { difference }\end{array}$} & \multirow{2}{*}{$\begin{array}{l}\text { Effect size } \\
\text { (Cohen's } d \text { ) }\end{array}$} \\
\hline & $M$ & $S D$ & $M$ & $S D$ & & & & \\
\hline \multicolumn{9}{|l|}{ Task 1} \\
\hline 2-elm & 94 & 18 & 95 & 21 & .837 & 657 & 1 & 0.09 \\
\hline \multicolumn{9}{|l|}{ Task 2} \\
\hline 2-elm & 39 & 22 & 98 & 59 & .897 & 1,510 & $58^{*}$ & 2.99 \\
\hline 3-elm & 43 & 10 & 113 & 61 & .735 & 1,179 & $70^{*}$ & 3.35 \\
\hline 4-elm & 39 & 12 & 137 & 73 & .900 & 1,496 & $100^{*}$ & 4.79 \\
\hline \multicolumn{9}{|l|}{ Task 3} \\
\hline 1-elm & 282 & 131 & 292 & 63 & .989 & 7,020 & 10 & 0.13 \\
\hline 2-elm & 250 & 59 & 342 & 121 & .391 & 13,196 & $92^{*}$ & 0.77 \\
\hline
\end{tabular}

Note. Group means and standard deviations are reported for controls and patients. Then, in order to address the psychometric confound question, we provide the reliability scores (standardized Cronbach's alpha; Cronbach, 1951) and the discrimination power (true score variances [TSV]. True score variance $[\mathrm{VAR}]=$ reliability $*$ observed score $\left.\mathrm{VAR}_{\text {controls }}\right)$ for the control group only, and the group differences as well as the effect sizes $($ Cohen's $d$; $d_{\text {Cohen }}=\left[M_{\text {control }}-M_{\text {patients }}\right] /\left[S D_{\text {pooled }}\right]$, with $S D_{\text {pooled }}=\operatorname{root}\left[\left(\right.\right.$ observed score $\mathrm{VAR}_{\text {controls }}+$ observed score $\left.\left.\left.\mathrm{VAR}_{\text {patients }}\right) / 2\right]\right)$ for each task and condition. One can clearly note that the pattern of group difference is not in the same ranked order as that observed for the TSV. Namely, the global (one-element [1-elm]) but not the segmented (two-element [2-elm]) condition of Task 3 has greater TSV than any condition in Tasks 1 or 2 but yields no significant group difference. Hence, the results suggest that the motor deficits in schizophrenia are not due to a generalized deficit but may be associated with a specific problem of planning action sequences through time. Results are reported for movements requiring subjects to sequence two elements (2-elm), three elements (3-elm), or four elements (4-elm).

$\alpha=.05$ 
Table 2

Intercorrelations Between Tasks and Conditions

\begin{tabular}{|c|c|c|c|c|c|c|}
\hline $\begin{array}{l}\text { Task \& } \\
\text { condition }\end{array}$ & 1 & 2 & 3 & 4 & 5 & 6 \\
\hline 1. Task 1: 2-elm & - & & & & & \\
\hline 2. Task 2: 2-elm & .13 & - & & & & \\
\hline 3. Task 2: 3-elm & .09 & .93 & - & & & \\
\hline 4. Task 2: 4-elm & .08 & .91 & .97 & - & & \\
\hline 5. Task 3: 1-elm & .07 & .15 & .17 & .17 & - & \\
\hline 6. Task 3: 2-elm & .01 & .35 & .36 & .29 & .32 & - \\
\hline
\end{tabular}

Note. This table presents the intercorrelation values for the various tasks and conditions in order to reveal discrimination among tasks that putatively measure different processes. The most significant correlations are found within Task 2 (boldfaced). One can note the close to null correlation values between Tasks 1 and 2, suggesting that these tasks very probably tap different cognitive processes. Results are reported for movements requiring subjects to sequence two elements (2-elm), three elements (3-elm), or four elements (4-elm).

order to readjust finger force to the changing forces at the fingerobject contact. Here, the mean time delay between the two successive motor elements was around $95 \mathrm{~ms}$, delay which suggests the intervention of long latency reflexes for the reactive control of precision grip (Johansson et al., 1994; Johansson \& Westling, 1987). Clinical studies have shown that these fast integrative mechanisms-which are too quick to permit voluntary-cognitive control-are very probably subcortical. Indeed, the somatosensory-driven control of precision grip (a) is not affected by TMS on the motor cortical regions (Macefield \& Johansson, 1994; Macefield, Rothwell, \& Day, 1996) and (b) is normal in X-linked Kallmann patients who have neurological deficits to the fast-conducting cortico-spinal system (Harrison et al., 2000). In the present study, we showed that the group mean time delays between two motor elements for triggered sequences were similar in patients with schizophrenia and in controls. These results support the hypothesis of preserved lower order (subcortical) integrative mechanisms in schizophrenia. In addition, a close examination of the force patterns during the 250-ms baseline measures (Figure 1) failed to show any evidence of tardive dyskinesia in our patients with schizophrenia. Overall, our results argue against an explanation of sequencing deficits in schizophrenia in terms of dysfunctional lower order sensorimotor processes.

In the second task (planned sequences), subjects were required to execute a series of planned motor sequences of increasing complexity. For the controls, the time delay between successive elements was shorter for planned $(40 \mathrm{~ms})$ than for triggered (96 $\mathrm{ms}$ ) sequences. This suggests that both the order and the timing of the motor elements were preprogrammed, and that sensory afferents were not used for the fluent execution of the planned sequences. Furthermore, as the interelement time delay did not increase with sequence complexity, one can hypothesize that no further online processing of the sensory information took place during the execution of the task (Sternberg et al., 1989). Overall, our results are in agreement with those results reported in timing studies, which suggest that the role of sensory information is not to initiate or execute motor sequences but, rather, to minimize synchronization errors that may occur between self-produced actions and external pacing signals (Billon et al., 1996).

A different pattern of results was revealed in our patients with schizophrenia. Their performance was characterized by interelement delays that were (a) longer for planned than for triggered sequences, (b) abnormally long compared with the controls, and (c) increased with sequence complexity. Overall, these data support the possibility that schizophrenia is characterized by a lack of fluency in the execution of planned sequences, that is, a higher order deficit in the temporal sequencing of multiple motor elements.

The fact that in patients the interelement time delay increased with sequence complexity might have suggested a difficulty in preplanning the entire sequence before its initiation; consequently, further processing might have been required during task execution. To further look into this possibility, we used a third task (context effect) for which subjects were required to perform a simple pointing act. Through instructions that emphasized either speed of starting or speed of completing the action, this movement was considered either a single-element (i.e., nonsequential) action or a two-element sequence action. In either case, we considered that preprogramming was required to react quickly to the auditory signal. Results revealed that when no sequencing was required, patients were as quick as controls in reacting to the auditory signal. When instruction indicated the need for sequencing, patients were significantly slower than controls (Figure 3). This finding argues against a general problem in motor planning but argues in favor of a specific problem in the fluent sequencing of action when planning is required. It is interesting to note that in the literature, many studies suggest general slowness in schizophrenia for manual reaction times (Cohen \& Servan-Schreiber, 1992; Sereno \& Holzman, 1995; for a review see Nuechterlein, 1977), but the selected tasks often required sequencing, for example, detect a white dot and then detect a blue dot and then press the switch as soon as the blue dot disappears (Zahn, Roberts, Schooler, \& Cohen, 1998). Hence, it is possible that slowness is not characteristic of patients with schizophrenia but arises in these patients because sequencing is necessary. Little mention of this task effect has been made in the literature, and it will be important to confirm this finding with additional studies.

Losing the capacity to coordinate multiple elements of a motor sequence within a global action, the preplanning phase is limited and consequently might be expected to leave the central nervous system in need of sensory feedback about the end of each motor element in order to trigger each successive element of the sequence. This explanation is consistent with the results reported in our patients with schizophrenia. Indeed, the interelement time delays for planned sequences (109 ms) were at least as long and often longer that those observed for triggered sequences $(95 \mathrm{~ms})$. 
Hence, for planned sequences, patients may have relied on the preserved sensorimotor lower order functions to initiate subsequent elements in both triggered and planned motor sequences, rendering the execution of the latter rather fragmented and nonfluent in appearance.

The patterns of results in the three contrasting tasks allow us to eliminate three possible alternative explanations for the reported motor impairments. First, it might be argued that the sequencing deficits could have been due to a difficulty in maintaining the focus of attention throughout the series of trials. However, in this case, the variability of the reaction and movement times would have been significantly increased in patients compared with controls, and this was not the case.

Second, it might be suggested that the abnormal pattern of results in the schizophrenic group resulted from general slowness. Against this, we note that patients exhibited movement and reaction times similar to those measured in controls (Tasks 1 and 3) yet still exhibited abnormal time delays in the planned sequences (Task 2).

Third, it might be argued that the deficits could have arisen from the effects of neuroleptic medication. We suggest that this is unlikely as the sequencing deficits were observed in patients with $(N=21)$ and without $(N=3)$ neuroleptic treatment. Furthermore, force patterns in Task 1 revealed an absence of Parkinson-like syndrome effect in all patients. However, given the small number of unmedicated patients we acknowledge the need for further replication studies with medication-free patients.

Last, one could argue that fluency deficits appeared in patients with schizophrenia in Task 2 because of its greater discriminating power. Indeed, the comparison of Tasks 1 and 2 is confounded with differential discriminating power (see TSVs and group differences in Table 1). However, the inclusion of Task 3, which was the task with the greatest discriminating power, gave us the means to show that patients performed as normal in a planned task that required no sequencing (1-elm, Task 3 ) but were impaired when sequencing was required (2-elm, Task 3; all conditions of Task 2). Hence, we suggest that motor deficits in schizophrenia are not due to a generalized deficit but may be associated specifically to a problem of planning action sequences through time.

In conclusion, our results confirm that even simple motor actions are impaired in schizophrenia as long as they require the sequencing of at least two elements. They furthermore indicate that this abnormality appears for higher order integration and not for the basic sensorimotor mechanisms that require minimal controlled processing. Because motor fluency is central to the control and execution of most commonly performed actions, a deficit in it can severely impact everyday life function. Hence, it will be important in future studies to determine to what extent the impairment revealed here for planned sequences is related to higher order cognitive functions (memory, attention) and clinical symptoms (Neumann \& Walker, 2003). Our previous results have suggested that the efference copy is preserved in schizophrenia when considering passive movements (Delevoye-Turrell, Giersch, \& Danion, 2002, 2003). Abnormalities were observed only for active ones. These results are coherent with those reported by Knoblich, Stottmeister, and Kircher (2004), which suggested that abnormal motor control is revealed in schizophrenia as soon as the movement becomes voluntary, that is, requires the subjects' attention (Frith, 2005). The data presented here in Task 3 suggest, however, that in schizophrenia, movement execution can be as fast as that observed in healthy controls even when voluntarily performed by the subject (1-elm condition, global). Abnormalities seem to be more specifically associated to the need to organize multiple subelements within a coherent and fluent motor plan (2-elm condition, segmented). Hence, one may ask: What occurs when multiple subelements are to be sequenced? Is the efference copy, itself, different for motor sequences than for unit actions? Or is the planning phase different because of the need to prepare for the smooth passage from one element of the sequence to the next? At the physiological level, this latter hypothesis would require good communication between brain areas concerned with initiating actions (frontal cortex) and sensory areas concerned with processing and integrating the consequences of actions (thalamus). There is some evidence in favor of abnormal connectivity between brain areas in schizophrenia (Fletcher, Frith, Grasby, Friston, \& Dolan, 1996; Fletcher, McKenna, Friston, Frith, \& Dolan, 1999). Hence, a fruitful line for future research in the cognitive neuropsychology of schizophrenia would be to explore whether abnormal connectivity between frontal and parietal regions via the thalamus is associated to fluency problems in the sequencing of planned (motor) actions.

\section{References}

American Psychiatric Association. (1994). Diagnostic and statistical manual of mental disorders (4th ed.). Washington, DC: Author.

Andreasen, N. C. (1999). A unitary model of schizophrenia. Bleuler's "Fragmented Phrene" as schizencephaly. Archives of General Psychiatry, 56, 781-787.

Billon, M., Semjen, A., Cole, J., \& Gauthier, G. (1996). The role of sensory information in the production of periodic finger-tapping sequences. Experimental Brain Research, 110, 117-130.

Caligiuri, M. P., \& Lohr, J. B. (1994). A disturbance in the control of muscle force in neuroleptic-naive schizophrenic patients. Biological Psychiatry, 35, 104-111.

Chapman, L. J., \& Chapman, J. P. (1973). Problems in the measurement of cognitive deficit. Psychological Bulletin, 79, 380-385.

Cohen, J. D., \& Servan-Schreiber, D. (1992). Context, cortex and dopamine: A connectionist approach to behavior and biology in schizophrenia. Psychological Review, 99, 45-77.

Crespo Facorro, B., Paradiso, S., Andreasen, N. C., \& O'Leary, D. S. (1999). Recalling word list reveals cognitive dysmetria in schizophrenia patients: A PET study. American Journal of Psychiatry, 156, 386-392.

Cronbach, L. J. (1951). Coefficient alpha and the internal structure of tests. Psychometrika, 16, 297-334.

Delevoye-Turrell, Y., Giersch, A., \& Danion, J.-M. (2002). A deficit in the adjustment of grip force responses in schizophrenia. NeuroReport, 13, 1537-1539.

Delevoye-Turrell, Y., Giersch, A., \& Danion, J.-M. (2003). Abnormal sequencing of motor actions in patients with schizophrenia: Evidence from grip force adjustments during object manipulation. American Journal of Psychiatry, 160, 134-141.

Delevoye-Turrell, Y., \& Wing, A. M. (2004). Adaptive behavior for an intended goal. In K. Lamberts \& R. Goldstone (Eds.), The handbook of cognition (pp. 130-157). Thousand Oaks, CA: Sage.

Flanagan, J. R., \& Wing, A. M. (1997). The role of internal models in motion planning and control: Evidence from grip force adjustments during movements of hand-held loads. Journal of Neuroscience, 17, 1519-1528.

Fletcher, P., Frith, C. D., Grasby P. M., Friston, \& Dolan, R. J. (1996). Local and distributed effects of apomorphine on fronto-temporal function in acute unmedicated schizophrenia. Journal of Neuroscience, 16 , 7055-7062. 
Fletcher, P., McKenna, P. J., Friston, K. J., Frith, C. D., \& Dolan, R. J. (1999). Abnormal cingulated modulation of fronto-temporal connectivity in schizophrenia. Neuroimage, 9, 337-342.

Frith, C. (2005). The neural basis of hallucinations and delusions. Comptes Rendus Biologies, 328, 169-175.

Gordon, A. M., \& Soechting, J. F. (1995). Use of tactile afferent information in sequential finger movements. Experimental Brain Research, 107, 281-292.

Harrison, L. M., Mayston, M. J., \& Johansson, R. S. (2000). Reactive control of precision grip does not depend on fast transcortical reflex pathways in X-linked Kallmann subjects. Journal of Physiology, 527, 641-652.

Johansson, R. S., Häger, C., \& Riso, R. (1992). Somatosensory control of precision grip during unpredictable pulling loads. III. Impairments during digital anesthesia. Experimental Brain Research, 89, 204-213.

Johansson, R. S., Lemon, R. N., \& Westling, G. (1994). Time-varying enhancement of human cortical excitability mediated by cutaneous inputs during precision grip. Journal of Physiology, 481, 761-775.

Johansson, R. S., Riso, R., Häger, C., \& Bäckström, L. (1992). Somatosensory control of precision grip during unpredictable pulling loads. I. Changes in load force amplitude. Experimental Brain Research, 89, 181-191.

Johansson, R. S., \& Westling, G. (1984). Roles of glabrous skin receptors and sensorimotor memory in automatic control of precision grip when lifting rougher or more slippery objects. Experimental Brain Research, $56,550-564$.

Johansson, R. S., \& Westling, G. (1987). Signals in tactile afferents from the fingers eliciting adaptive motor responses during precision grip. Experimental Brain Research, 66, 141-154.

Knight, R. A., \& Silverstein, S. M. (2001). A process-oriented approach for averting confounds resulting from general performance deficiencies in schizophrenia. Journal of Abnormal Psychology, 110, 31-39.

Knoblich, G., Stottmeister, F., \& Kircher, T. T. J. (2004). Self-monitoring in patients with schizophrenia. Psychological Medicine, 34, 1561-1569.

Lecrubier, Y., Sheehan, D. V., Weiller, E., Amorim, P., Bonora, I., Sheehan, K. H., et al. (1997). The Mini International Neuropsychiatric Interview (M.I.N.I.), a short diagnostic interview: Reliability and validity according to the CIDI. European Psychiatry, 12, 224-231.

MacDonald, A. W., \& Kang, S. S. (2006). Cassandra's calculations: Simulation studies of the psychometric confound. In D. P. French (Ed.), Schizophrenic psychology: New research. Hauppauge, NY: Nova Science Publishers.

Macefield, V. G., \& Johansson, R. S. (1994). Electrical signs of cortical involvements in the automatic control of grip force. NeuroReport, 5, $2229-2232$.

Macefield, V. G., Rothwell, J. C., \& Day, B. L. (1996). The contribution of transcortical pathways to long-latency stretch and tactile reflexes in human hand muscles. Experimental Brain Research, 108, 147-154.

Miller, M. B. (1995). Coefficient alpha: A basic introduction from the perspectives of classical test theory and structural equation modeling. Structural Equation Modeling, 2, 255-273.

Müller, F., \& Dichgans, J. (1994). Dyscoordination of pinch and lift forces during grasp in patients with cerebellar lesions. Experimental Brain Research, 101, 485-492.

Neumann, C. S., \& Walker, E. F. (1999). Motor dysfunction in schizotypal personality disorder. Schizophrenia Research, 38, 159-168.

Neumann, C. S., \& Walker, E. F. (2003). Neuromotor functioning in adolescents with schizotypal personality disorder: Associations with symptoms and neurocognition. Schizophrenia Bulletin, 29, 285-298.

Nowak, D. A., Glasauer, S., Meyer, L., Mai, N., \& Hermsdorfer, J. (2002). The role of cutaneous feedback for anticipatory grip force adjustments during object movements and externally imposed variation of the direction of gravity. Somatosensory Motor Research, 19, 49-60.

Nuechterlein, K. H. (1977). Reaction time and attention in schizophrenia: A critical evaluation of the data and theories. Schizophrenia Bulletin, 3, 373-428.

Nunnaly, J. (1978). Psychometric theory. New York: McGraw-Hill.

Rothwell, J. C., Traub, M. M., Day, B. L., Obeso, J. A., Thomas, P. K., \& Marsden, C. D. (1982). Manual motor performance in a deafferented man. Brain, 105, 515-542.

Sereno, A. B., \& Holzman, P. A. (1995). Antisaccades and smooth pursuit eye movements in schizophrenia. Biological Psychiatry, 37, 394-401.

Sternberg, S., Knoll, R. L., Monsell, S., \& Wright, C. E. (1989). Motor programs and hierarchical organization in the control of rapid speech. Phonetica, 45, 175-197.

Strauss, M. E. (2001). Demonstrating specific cognitive deficits: A psychometric perspective. Journal of Abnormal Psychology, 110, 6-14.

Sullivan, E. V., Fama, R., Shear, P. K., Cahn-Weiner, D. A., Stein, M., Zipursky, R. B., \& Pfefferbaum, A. (1994). Motor sequencing deficits in schizophrenia: A comparison with Parkinson's disease. Neuropsychology, 15, 342-350.

Turrell, Y., Li, F. X., \& Wing, A. M. (2001). Estimating the minimum grip force required when grasping objects under impulsive loading conditions. Behavior Research Methods, Instruments, \& Computers, 33, 38 45.

Vrtunski, P. B., Simpson, D. M., \& Meltzer, H. Y. (1989). Voluntary movement dysfunction in schizophrenics. Biological Psychiatry, 25, $529-539$.

Wiser, A. K., Andreasen, N. C., O’Leary, D. S., Watkins, G. L., Ponto, L. L. B., \& Hichwa, R. D. (1998). Dysfunctional cortico-cerebellar circuits cause "cognitive dysmetria" in schizophrenia. NeuroReport, 9, 1895-1899.

Zahn, T. P., Roberts, B. R., Schooler, C., \& Cohen, R. (1998). Manual and saccadic reaction time with constant and variable preparatory intervals in schizophrenia. Journal of Abnormal Psychology, 107, 328-337.

Received July 6, 2005

Revision received September 13, 2006 Accepted September 18, 2006 